IRA-International Journal of Technology \& Engineering ISSN 2455-4480

Proceedings of the

International Conference on Science \& Engineering for Sustainable Development (2017)

Pg. no.113-121

Published by: Institute of Research Advances https://research-advances.org/index.php/IRAJTE

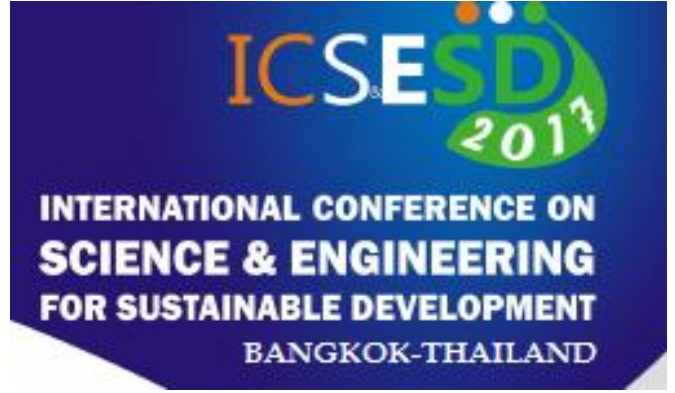

\title{
Validity Testing of a Simplistic Design Version of a Kinematic Arm for Postural Measurement of Human Body Segments (May 2017)
}

\author{
Vinod U Datir**1, B. N. Lahari ${ }^{2}$, Vijay V Kale ${ }^{3}$ \\ 1,2 Jadavpur University, Kolkata(WB), India \\ ${ }^{3}$ Prof.Ram Meghe Institute of Technology \& Research, Badnera, Amravati, India.
}

Type of Review: Originality Check \& Peer Review under the responsibility of the Scientific Committee of the Conference and The Institution of Engineers (India). DOI: http://dx.doi.org/10.21013/jte.ICSESD201712

\begin{abstract}
How to cite this paper:
Datir, V., Lahari, B., Kale, V. (2017). Validity Testing of a Simplistic Design Version of a Kinematic Arm for Postural Measurement of Human Body Segments (May 2017). Proceedings of the International Conference on Science \& Engineering for Sustainable Development (2017), 113121. doi: http://dx.doi.org/10.21013/ite.ICSESD201712
\end{abstract}

(C) International Conference on Science \& Engineering for Sustainable Development\& The Institution of Engineers (India).

\section{(c) EY-NC}

This work is licensed under a Creative Commons Attribution-Non Commercial 4.0 International License subject to proper citation to the publication source of the work.

Disclaimer: The conference papers as published by the Institute of Research Advances (IRA) are the views and opinions of their respective authors and are not the views or opinions of the IRA. The IRA disclaims of any harm or loss caused due to the published content to any party. 


\section{ABSTRACT}

In all engineering industries complaints about back pain and low back injuries are very high. Major factor for such back injuries are associated with Load handling tasks. In industries type of such lifting activities in percentage is to extend $77 \%$ and bending activity up to 56\%.According to US department of labour report back injuries account to be $20 \%$ of all injuries and $25 \%$ of annual worker compensation goes for such injuries.

So one of the important areas of Kinematic And kinetic measurements are useful is load lifting. Different methods have been evolved for carrying out Kinematic measurements, selection depend upon type of study, type of kinematic parameter to obtained merits and demerits of the methods and cost of study.

Need of alternative methods which satisfy the study requirements and also compensate the cost leads to a development of "Kinematic Arm" In one of study made by Belli et al(1992) Developed Kinematic arm for three dimensional recording of human movements. They Validate and tested successfully the device. Error values for static quasi-static human motions are less than tracking system and film or video analysis ,Also device is five times cheaper than above two well known methods.

Based on the guidelines of study by Belli et al a Kinematic arm has been designed and fabricated for validation testing of positional measurements of human body segments subject to different postures during load lifting.

The Kinematic arm consists of four light rigid pipe linked together by three joints. One end of arm is connected to fixed reference point while other end can move freely in the three spatial directions. the free movement is allowed in all spatial directions by orientation of the axes of the three revolute joints. At each joint a potentiometer (multi turn 50 Kilo-ohm) is mounted and connected to an electronic interface to measure the angle between two link elements involved. Three potentiometers are connected in series and fed a constant current from the circuit. Output from potentiometer is amplified and measured and calibrated in terms of angles. Angles al $(t), a 2(t), a 3(t)$.measured and by appropriate trigonometric equations can calculate instantaneous position. Equation for velocity and acceleration have been established in order to evaluate the exact performance of kinematic arm for positional measurements

Kinematic arm is tested for the static and quasi-static conditions for different body postures successfully. Results obtained are within the range of theoretical estimation of errors.

Keywords: Industry load handling, Kinematic measurement, Kinematicarm, Positional Measurements of human body segments ,static and quasi-static validation.

\section{Introduction}

IN all Engineering industries including manufacturing, complaints about back pain and lower back pain are very high. The estimate according to the US department of labor report (1982), is that the back injuries account to $20 \%$ of all injuries and illness at workplace, and $25 \%$ of the annual worker compensation payments goes for such injuries

The major factor for such back injuries is associated with "load handling "tasks. The type of activities and type of movement in percentage associate with such tasks in industries reveal that lifting as an activity is to be extend of $75 \%$ and bending as a movement posture, is as high as $56 \%$. 
So one of the important areas of Kinematic and kinetic measurements are useful is load lifting. Different methods have been evolved for carrying out Kinematic measurements, selection depend upon type of study, type of kinematic parameter to obtained merits and demerits of the methods and cost of study.

Need of alternative methods which satisfy the study requirements and also compensate the cost leads to a development of "Kinematic Arm" In one of study made by Belli et al(1992) Developed Kinematic arm for three dimensional recording of human movements. They Validate and tested successfully the device. Error values for static quasi-static human motions are less than tracking system and film or video analysis, Also device is five times cheaper than above two well known methods.

\section{Kinematic Measurement of Human Motions}

From a mechanical standpoint, their functioning of human body may be viewed as composed of motion of a series of links as rigid segments, powered by muscles and connected at pivot points, the joints. In this model all activity is made-up of interactive movements, as illustrated by angular displacements of straight lines connecting the axis of rotation of the various segments.

From the above view point the human can be studied though kinematic measurement by applying the linkage concept. The human body can be modeled as connected links of head, neck and trunk( crown to hip joint),upper extremity( shoulder to forearm) and lower extremity( hip joint to soles of feet).Diagrammatically it is shown in fig 1.1.The link 1 consist of series of connected links, so also link 2 And link 3, while studying human motions laboratory model can be prepared with the help of linkage concept and relative motion between links can be studied. For studying upper extremity motions which consist of shoulder-elbow-forearm this can be modeled as a series of inter connected links, shown in Fig1.2.

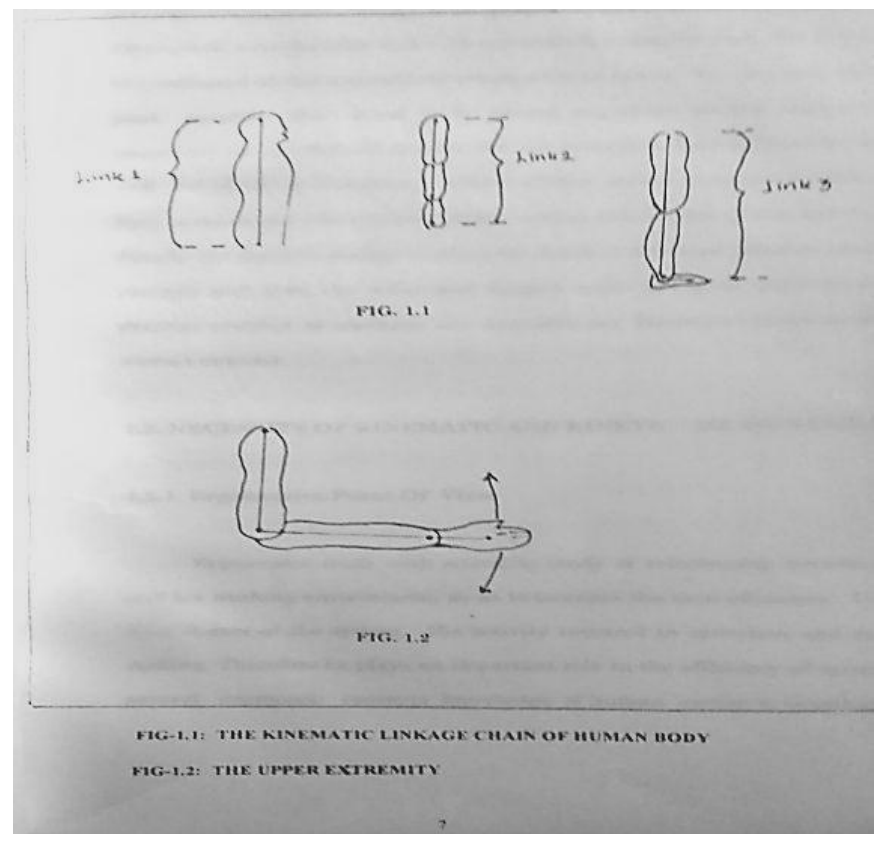

\section{Methods For Human Motion (Kinematic) Measurements}

Different methods have been evolved for carrying out kinematic measurement. Selection of particular method depends upon the study to be made and which kinematic parameters are required to obtain. It also depends on relative merits and demerits of the methods. Some of the practical methods which are commonly used for kinematic measurements are 
1. Film or video analysis

2. Tracking system

3. Accelerometer transducers

4. Force plate or force platform

1. Film or Video Analysis

This is the method mostly used for direct recording of human motions and other for obtaining data from recording. One method (Cochrn1982)uses high speed 16mm movie camera to film the subject in different planes. After this the film is developed and each frame is analyzed .Angular displacement and positions are calculated by linked computer. This method is labour intensive and time consuming . This problem can solve by tracking method

\section{Tracking Method}

Tracing method uses infrared-reflecting or emitting sensors as an adjunct to tracking. The Track III System can measure position with a translational accuracy $1 \mathrm{~mm}$ and rotational accuracy of $1^{\circ}$.It is basically optoelectronic position measurement system.

By this method problem of digitization can overcome but on-line computation not possible.

\section{Accelerometry or Acceleration transducer Method}

In both above kinematic measurement methods instantaneous positions are first measured and then $b$ time-differentiating this information velocity and accelerations are calculated. But as differentiation is noise-enhancing process, the alternative way to obtain kinematic parameters is to measure the instantaneous acceleration or force and to time integrate this information to obtain velocity and displacement. This approach is used in accelerometry and force platform methods.

But use of these methods gives rise to different problem. It requires integration constant that means position and velocity must be independently measured.

\section{Need Of Alternative Method}

The inference that can be drawn from above discussion that every method have its merits and demerits. The comparative studies made by different researchers on kinematic measurement of three dimentional human motions reveal that the efforts to compare parameters obtained by all discussed methods have been done only with the measurements dealing with mechanical system which is economical also. Therefore there is a need to develop simple, reliable and fast responding device for recording and computing displacement, velocity and accelerations.

Need of alternative methods which satisfy the study requirements and also compensate the cost leads to a development of "Kinematic Arm" In one of study made by Belli et al(1992) Developed Kinematic arm for three dimensional recording of human movements. They Validate and tested successfully the device. Error values for static quasi-static human motions are less than tracking system and film or video analysis, Also device is five times cheaper than above two well known methods.

\section{Objective And Scope Of Work}

\section{The Objective}

The cause of back pain is appearance of very large reaction forces at particularly the lumber vertebrae transmitted through different other joints of human skeleton .in this process the muscles are overloaded. A combined knowledge of kinematic, kinetic and energetic is necessary for this understanding.

The objective of this work is however limited to kinematic study of human three dimensional motions and to develop, validate a simple, reliable mechanical device i.e "Kinematic Arm" 


\section{The Scope}

The present scope as

1. To develop and fabricate the kinematic arm

2. To arrange a compatible electronic power package to drive, monitor and measure reliably the signals from sensors.

3. To validate the device for positional measurement of human body segment motions in both static as well as quasi-static positions

4. Statically establish the acceptability of errors in such measurements

\section{Design of Kinematic Arm}

\section{Description Of Kinematic Arm}

Based on the guidelines of study by Belli et al a Kinematic arm has been designed and fabricated for validation testing of positional measurements of human body segments subject to different postures during load lifting.

The Kinematic arm consists of four light rigid pipe linked together by three joints. One end of arm is connected to fixed reference point while other end can move freely in the three spatial directions. the free movement is allowed in all spatial directions by orientation of the axes of the three revolute joints. The first and second joints give movements of link in $X-Z$ plane while the third joint is oriented in such a way that its axis always moves parallel to the $X-Z$ plane so that the free moving end can move in all the three(X,Y,Z)spatial directions. At each joint a potentiometer (multi turn 50 Kilo-ohm) is mounted and connected to an electronic interface to measure the angle between two link elements involved. Three potentiometers are connected in series and fed a constant current from the circuit. Output from potentiometer is amplified and measured and calibrated in terms of angles. Angles a1(t),a2(t),a3(t) can bemeasured and by appropriate trigonometric equations can calculate instantaneous position.(as indicated in Fig2.1) Equation for velocity and acceleration have been established in order to evaluate the exact performance of kinematic arm for positional measurements

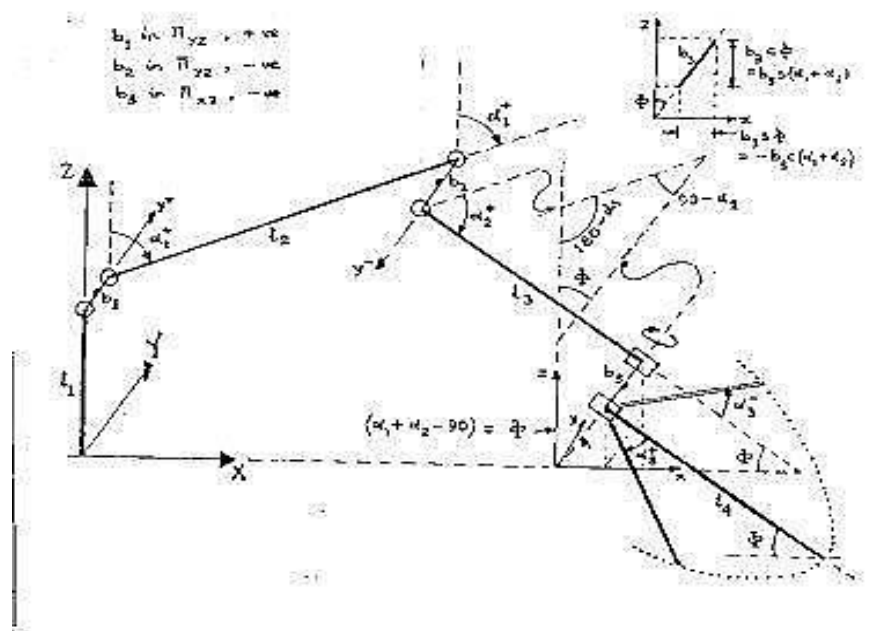

Figure2.1 Kinmatic Arm 


\section{Specification}

With reference to study of A.Belli et al(1992) a general specifications of the Kinematic arm are as

- Joints and links elements were made of light material

- The maximum total mass of kinematic arm including joints,sensors ,wires and segments was about $900 \mathrm{gm}$

- The best combination is the compromise between arm with short length element and an arm with large link elements. Short segment arm gives accurate measurement and long segment allows large movement of measuring end.

\section{Identification Of Design Parameters}

Due to no availability of optoelectronic transducers helical potentiometers are used as an alternative. For link elements of kinematic arm light, rigid aluminium tubes of different lengths are used by measuring their mass, length and by arranging different combinations suitable combination finalized. The joint were designed to facilitate easy axis orientation and mounting of potentiometer. for this two separate block were designed pot block and knob block. The whole design of kinematic arm should be such that it allows free orientation of axis and calibration

From above analysis common parameters identified in design of kinematic arm are

1.Size of link elements

2. Design of joints

3. Base of kinematic arm

4.Identification of best suited combination

\section{Electronic Interface}

Description of kinematic arm indicates it is required to measure the angle between two adjacent links. So by obtaining the angle between all three joints instantaneous position of free moving end can calculate. There is necessary to mount sensor at each joint. Also suitable electronic circuit necessary to give input to sensor and to read output signal from sensor.

At each joint a potentiometer (multi turn 50 Kilo-ohm) is mounted and connected to an electronic interface to measure the angle between two link elements involved. Three potentiometers are connected in series and fed a constant current from the circuit. Output from potentiometer is amplified and measured and calibrated in terms of angles. Angles a1 (t),a2(t),a3(t).measured and by appropriate trigonometric equations can calculate instantaneous position.

Electronic interface consist of

1.Power supply

2.Constant current sink

3.Potentiometric system

4.Amlifiers(3 such)

\section{Principal of Operation Of The Circuit}

Circuit operates on the principal as any change in position of potentiometer changes its resistance which in turn changes in voltage across the potentiometer terminals. This difference voltage across the terminal is amplified to get the difference in position as output which can read by digital multimeter. This output position for each pot can be recorded for the different position of pot and calibrated in terms of angles. 
To the potentiometric system pots are connected in series and a constant current is supplied from constant current sink.

\section{Validation Testing Of Kinematic Arm For Positional Measurement}

In order to know the kinematic arm performance for positional measurement of human body segments in different postures, it was tested and validated for static and quasi-static conditions.

While testing and validating kinematic arm instantaneous position of free moving end can calculate by developing the appropriate trigonometric equations

On the guidelines of A.Belli et al(1992) trigonometric equations were developed for kinematic arm mentioned in study. Instataneous position of free moving end can calculate according to following equations

$$
\begin{gathered}
x(t)=l_{2} \sin \left(\alpha_{1}(t)\right)+l_{3} \sin \left(\left(\alpha_{1}(t)\right)+\alpha_{2}(t)\right)+l_{4} \cos \left(\alpha_{3}(t)\right) \sin \left(\alpha_{1}(t)\right) \sin \left(\alpha_{2}(t)\right)+\alpha_{2}(t) \\
-b_{3} \cos \left(f \alpha_{1}(t)+\alpha_{2}(t)\right) \\
y(t)=b_{1}+b_{2}+l_{4} \sin \left(\alpha_{3}(t)\right) \\
z(t)=l_{1}+l_{2} \cos \left(\alpha_{1}(t)\right)+l 3 \cos \left(\alpha_{1}(t)+\alpha_{2}(t)\right)+l_{4} \cos \left(\left(\alpha_{3}(t) \cos \left(\alpha_{1}(t)+\alpha_{2}(t)\right)+b_{3} \sin \left(\alpha_{1}(t)\right.\right.\right. \\
\left.+\alpha_{2}(t)\right)
\end{gathered}
$$

Where

$\mathrm{x}(\mathrm{t}) . \mathrm{y}(\mathrm{t}), \mathrm{z}(\mathrm{t})=$ Position in $\mathrm{X}, \mathrm{Y}, \mathrm{Z}$ directions $(\mathrm{m}) ; \quad \mathrm{l}_{1}, \mathrm{l}_{2}, 3, \mathrm{l}_{4}=$ length of segments $(\mathrm{mm})$ which are $\mathrm{l}_{1}=170 \mathrm{~mm}, \mathrm{l}_{2}=400 \mathrm{~mm}, \mathrm{l}_{3}=300 \mathrm{~mm}, \mathrm{l}_{4}=300 \mathrm{~mm}$;

$\mathrm{b}_{1}, \mathrm{~b}_{2}, \mathrm{~b}_{3}=$ clearance between pot block and knob block of first second and third joint $\mathrm{b}_{1}=29 \mathrm{~mm}, \mathrm{~b}_{2}=$ $31 \mathrm{~mm}, \mathrm{~b}_{3}=-31 \mathrm{~mm}$;

$\alpha_{1}(t), 2(t), \alpha_{3}(t)=$ angles of the different joints

Validation testing of kinematic arm includes

1. Calibration

2. Testing of kinematic arm

3. Validation of kinematic arm

\section{Calibration}

Output of potentiometer in terms of voltage, necessary calibration is required to read the output in terms of degree (angle).For this scale is calibrated by taking different positions of pot and by noting the corresponding output and positions. Seperate scale prepared for each potentiometer.

\section{Testing of Kinematic Arm}

Kinematic arm tested for

a. Static condition

b. Quasi-static condition

a. Testing for Static condition

Testing of kinematic arm for static condition was carried out by arranging number of possible positions of kinematic arm relative to $\mathrm{X}, \mathrm{Y}, \mathrm{Z}$ directions in space and measuring the distance of moving end from fixes end(reference length).

For number of possible combinations of kinematic arm about 1045 measurements were obtained. By this way all possible positions of arm were tested.

In another method testing of kinematic arm was carried out by measuring the reference length repeatedly 10 times by arranging kinematic arm in different positions relative to measured direction. By these way measurements for different lengths was obtained. 
b. Testing of Kinematic Arm for Quasi-static Condition

For this test the free end of the arm needs tobe rotated in some plane. The orientation of planes can be any of three directions. Two extreme possibilities are there, The plane rotation of free end can lie either parallel to $\mathrm{X}-\mathrm{Z}$ plane or perpendicular to the $\mathrm{X}-\mathrm{Z}$ plane.

On cycle ergometer, subject was asked perfom cycling at slow speed at no load condition and the free moving end of arm attach to the ankle joint of subject.Reading was recorded for four positions of ankle joints (Fig;3.1)

\section{Result and Discussion}

The error is defined as the difference between the reference length and the value computed from the kinematic arm. For the repeated measurements of reference length were obtained. The absolute value $(\mathrm{mm})$ and relative value in percentage of measured value, mean error, standard deviation of the error(SDE) and of maximal error calculated The relative value of SDE is called as signal to noise ratio.

\section{Static Validation Results}

When the measurements are carried by "kinematic arm " for both first and second methods the maximal error were found to be 3,6,7,9 and $-8,-18$ respectively.

The maximal error theoretically depends upon arm segment lengths .In present case resolution of potentiometer is equivalent to $0.5 "=0.0087 \mathrm{rad}$, hence theoretical error introduced by one joint is $8.7 \mathrm{~mm}$. The mechanical tolerances on joints and links and cross sensitivity of potentiometer explain that measured maximal error were higher but in the same range of theoretical values.

2 Quasi-static Validation Results

During the test different configuration of lower limb of human subject was obtained .

Angle sustained by ankle at different configuration of lower limb of ankle joint is obtained and cross check of angle made for two positions of lower limb.

While obtaining the different confriguration change in length of foot during flexion and extension was considered. So from the data it is clear that angles obtained during flexion and extention of foot at ankle joint are nearby equal the angles obtained from photograph. Hence performance of kinematic arm for quasi-static condition is validated

\section{Conclusion}

From the testing and validation of kinematic arm for static and quasi-static conditions a general conclusions can be drawn

1. Static performance of kinematic arm is successfully tested and validated. Results obtained are within the range of theoretical estimation of error

2. Performance of kinematic arm for quasi-static condition is also successfully tested and validated for segments in different postures. 


\section{Acknowledgment}

The author express his sincere thanks to his esteemed teacher and the guide Dr B.N.Lahari Reader in Production Engineering department,Jadavpur University,Kolkata .Author also thanks to Prof.Tiberwalla,School of Bio-Engg. And Science,J.U.

\section{References}

[1] Halender M," A Guide to the Ergonomics of Manufacturing", East-West press/Taylor and Francis , 1995

[2] Hamill J.And KnutzenK,"Bio-Mechanical Basis of Human movement",Balada D.(Ed), Williams and Wilkins, 1995USA: Abbrev. of Publisher, year, ch. $x$, sec. $x$, pp. $x x x-x x x$.

[3] Cocharn G.V.B." A primer of Orthopaedic Bio-mechanics", Churchill Living Stone Pub.,Newyork, 1982

[4] Greory B.A.," An Introduction to Electrical Instrimentation",Unwin Brother(Eds), Ihe Macmillan Press ltd.W.-K.Chen,LinearNetworksandSystems.Belmont, CA:Wadsworth, 1993, pp. $123-135$.

[5] Apkarian J.,Naumann,J.,Cairns B. “A Three dimentional kinematic and Dynamic model of the lower limb",. Journal of Biomechanics, vol.22 No2,pp.143-155,1989.

[6] Belli A.,Rey S.Bonnefoy and Lacour J.R. "A simple device for kinematic measurement of human movement”,Journal of Ergonomics,vol.35,No.2,pp.177-186,1992

[7] Ladin Z.,Flowers W.C.,Messner W.," A Quatative comparisionof a Positional Measurement System and Accelerometry",Journal of Bio-mechanics,vol.22,pp.295-308,1980

[8] Smidt G.L.,Deusinger R.H.,Arora J.,Albright J.P.,"An Automated Accelerometry System for Gait Analysis"Journal of Bio-mechanics,vol.10,pp367-375,1977

[9] Kram R. and Powess A.J.," A Trademill-mounted Force platform”,Journal of Applied Physiology,vol.67,pp.1692-1698,1989.

[10] Inernational Labour Organisaton. "Kinetic Methods of manual Handling”, Occupational Health series,No19, GenevaName of 